\title{
The Influence of the Focus on the Counter-Propagating Optical Trapping
}

\author{
Liang Zhu ${ }^{1}$ FuLi Zhao ${ }^{1 a}$ \\ ${ }^{1}$ State Key Laboratory of Optoelectronic Materials and Technologies, Sun Yat-sen University, \\ Guangzhou, 510275, P. R. China.
}

Keywords: Counter-propagating optical trapping, focus separation, micron sized polyethylene particles,

\begin{abstract}
In our research, compared to conventional counter-propagating optical trapping setup, another CCD is used to help detect the focus in the XZ plane and thereby improving accuracy of the setup. Then we use this setup to accurately regulate three types of focus in counter-propagating dual beam optical trapping. Finally, some micron sized polyethylene particles are utilized to demonstrate the difference of these three types of focus. Therefore, we believe our work is beneficial to help explore the problem in counter-propagating optical trapping.
\end{abstract}

\section{Introduction}

Optical trapping is an intriguing area in Physics and Biology since it can help to move some micrometer and nanometer particles or blood red cells. In 1970, Ashkin proposed a method which is now called single optical trapping or optical tweezer to manipulate micron sized particles in 2-D [1] and attracts an increasing number of researchers to concentrate on this field [2-4]. Due to the existence of radiation pressure which could prevent the manipulation of the particles in 3-D, researchers begin to pay attention to some approaches in order to cancel the influence of the radiation pressure. In 2002, the Tatarkova's group implemented their experimental setup which is called counter-propagating optical trapping to cancel the radiation pressure [5]. They used the beam with the same intensity in each side so that in the sample area the radiation pressure can be cancelled to obtain better manipulation of particles in 3-D. After the emergence of some technology including spatial light modulator, the researches can easily change the wavefront and thereby receiving more and more new methods of optical trapping .For example. In 2001, Allen used LG which has the orbital angular momentum to rotate the larger calcite and small particles [6], In 2010, Alpmann's group used the Mathiu beam to assemble some elaborate particle micro structures in 3D[7]. In 2011, Mike Woerdemann and his partners utilized Ince-Gaussian beams to manipulate some silica spheres [8].

Even though a lot of papers and phenomena have been found in the area or optical trapping and counter-propagating optical trapping, there are still some problems. In the paper of Tauro in 2010, the Tauro's group introduced the effect of different focus separation like fig 1 [9]. Tauro pointed out that two diverging beams like fig 1a can create stable optical trapping but the low axial intensity gradient of the far-field beams leads to low axial stiffness and sluggish axial motion. When it comes to the fig1b, Tauro indicated that this setup can improve the stiffness of the optical trapping but it may become unstable when they used the objectives with lower NA. As for the fig 1c, Tauro

a)Author to whom correspondence should be addressed. Tel: 86-020-84112582. Electronic mail: stszfl@mail.sysu.edu.cn

considered that this setup would produce unstable optical trapping.
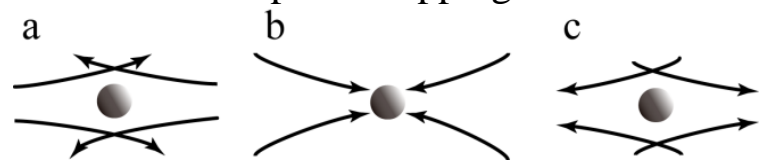

Fig1 a. Conventional stable CP-trapping of diverging beam b. Overlapping focus c. Converging beams with focus oppositely positioned compared to a

As we know, some researchers like Jones would like to use the counter-propagating optical trapping similar as fig1a [10], someone like Rkiouak used fig1b [11] and others including Yong-Le 
Pan [12] used fig1c. However, in their papers they did not point out why they rule out the other two focus modes. Therefore, in this paper, we focus on these three different types of focus. Firstly, we try to improve the experimental setup of the counter-propagating optical trapping in order to adjust the focus more accurately, and then we manipulate some micron sized polyethylene particles in these three ways in order to compare the advantages of each one.

\section{Samples preparation}

To achieve the stable optical trapping, the size of particles in the experiment of optical trapping are comparable to the size of the focus. In this case, we need to prepare our micron sized polyethylene particles nearly in 3 microns which is approximately the size of the Gaussian beam waist when we use the $40 \mathrm{X}$ objectives in our experiment. Consequently, some ten micron sized polyethylene rods are grinded into smaller micron particles with the size of 3 or 4 microns. The SEM of the polyethylene rods and particles are in the following fig 2.
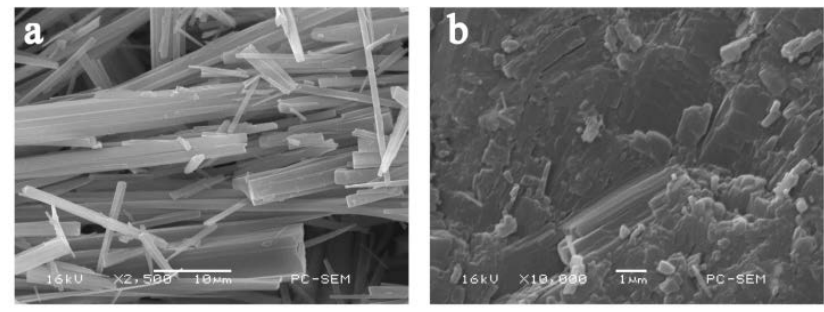

Fig 2 SEM of the polyethylene rods and particles

\section{Counter-propagating dual-beam optical trapping}

The representation of the optical system of conventional C-P dual-beam optical trapping is shown in fig 3a. The particles were trapped using the Picosecond laser operating at 532nm. We used Picosecond laser in our experiment because we would like to compare the results of the continuous wave laser with the Picosecond and Femtosecond laser in our future works. The aperture in the experiment was used to enable the size of the beam to overfill the back apertures of the $40 \mathrm{x}$ objectives. The attenuator on the left side was used to adjust the power of the beam in order to keep balance of the power from both side so that we can really cancel the effect of the radiation pressure to obtain stable optical trapping. The laser beam power for the optical trapping were 20mw on each side of the C-P dual-beam optical trapping. The filter prevented the 532nm laser beam and LED illumination enabled us to gain the image of the particles. In this conventional C-P dual beam optical trapping setup, the CCD was required to image the picture of YZ plane, which can help the researchers to adjust the distance of the focus. However, in our experiment like fig $3 \mathrm{~b}$, we use another CCD to image the XZ plane of the focus in order to control the focus more precisely. The 20x objective here acted as an condenser. In this setup of the experiment, we can use CCD to measure the beam waist of the Gaussian beam of each side which is nearly 4 microns. The size of the beam waist can help us to choose the particles in order to have stable optical trapping. 


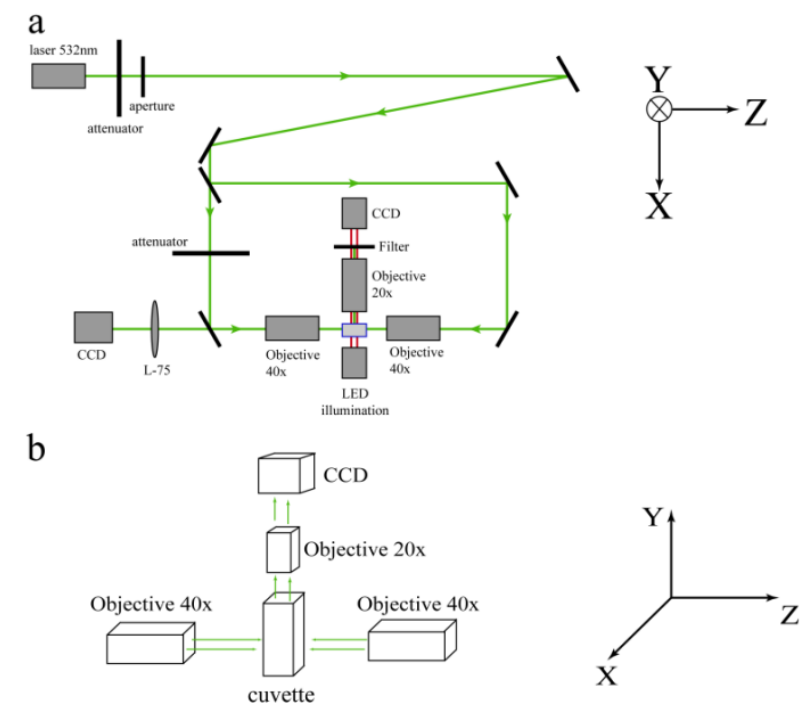

Fig 3 Experimental setup of Counter-Propagating dual-beam optical trapping a. conventional setup of C-P dual beam optical trapping b. Another CCD to detect the XZ plane of the focus.

\section{The focus of the C-P dual beam optical trapping}

After preparing the setup for the experiment, we control the focus of each side in order to make them overlap by adjusting the objectives. we regulate the beam power of each side to enable them to become $20 \mathrm{mw}$ by adjusting the attenuators. The images of the focus of the counter-propagating dual beam optical trapping is in the fig 4

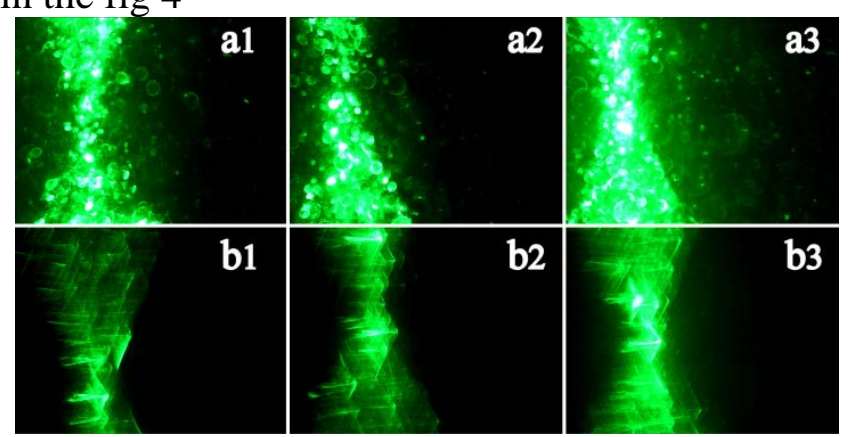

Fig 4 The image of the focus of the C-P dual-beam optical trapping using CCD. a1, a2, a3 are the images of the focus of the YZ plane,a1 is the focus of the left beam,a2 is the focus of the right beam, a3 is image of overlapping two focus, b1, b2, b3 are the images of the focus of the XZ plane. b1 is the focus of the left beam, b2 is the focus of the right beam, b3 is image of overlapping two focus. In the fig a1, a2, b1, b2 of the focus, we can see the beam have good Gaussian mode even though the existence of the light scattering. In the fig a3 and b3, we overlap this two Gaussian beam and obtain the situation of C-P dual-beam optical trapping we have discussed in fig1b. Then we adjust the objectives of each side to generate the focus separation like fig1a,fig1b and fig1c,respectively. After accurately controlling the focus separation, the experiment of optical trapping of the polyethylene particles is carried out.

\section{Results}

The results of the C-P dual-beam optical trapping of some polyethylene particles are shown bellow: 


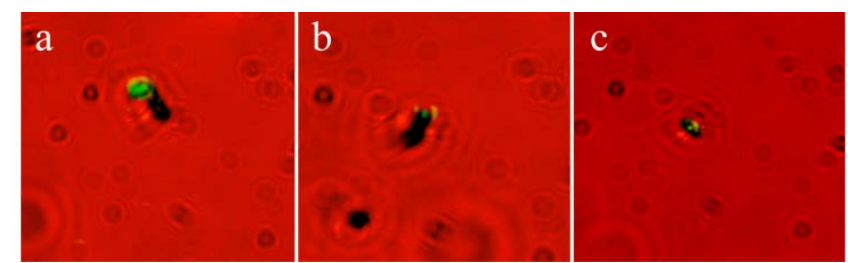

Fig 5 C-P dual-beam optical trapping of some polyethylene particles,

a) The optical trapping with the focus separation like fig 1a , b) the focus like fig1,c)the focus like fig1c.

In these three situations of the C-P optical trapping, we all have the phenomena of optical trapping. In the fig5, some particles are trapped near the focus of the Gaussian because of the optical gradient force. Moreover, due to the disappear of the radiation pressure because of the similar intensity of each side of the Gaussian beam, we can manipulate the particles in 3-D with the 40x objectives.

However, in the first case of the focus in fig5a, we can trap the polyethylene particles nearly 20 minutes and it is more stable than the other two. In the fig 5b, even though this overlapping focus has stable optical trapping, the particles will more easily float away when there is a little deviation from the focus. In this case, the overlapping focus in the fig $1 \mathrm{~b}$ have less work distance or trapping area than the other two. In the fig $5 \mathrm{c}$, this converging beam can also trap some particles near their focus, but it can't trap the particles stable and deviate the trapping area easily just in two minutes.

\section{Conclusion}

In our research, firstly, we use another CCD in other experimental setup to help adjust the focus of the counter-propagating dual beam optical trapping to enable it accurately in both $\mathrm{YZ}$ and $\mathrm{XZ}$ planes. Additionally, we experimentally discuss the difference of the three types of the focus of the counter-propagating dual beam optical trapping and use them to trap some micron sized polyethylene particles. We figure out that the diverging beam of the C-P optical trapping can trap the micron sized polyethylene particles longer and more stable than the other two when we use the $40 \mathrm{X}$ objectives. Therefore, we believe our work is helpful for the researchers to use the counter-propagating dual beam optical trapping.

\section{Acknowledgments}

This work was supported by the National Natural Science Foundation of China (10574165, 11274397), the Government of Guangdong Province for NSF

\section{References}

[1]. P. A. Ashkin, “Acceleration and trapping of particles by radiation pressure,” Phys. Rev. Lett. 24(4), 156-159 (1970).

[2] A. Ashkin and J. M. Dziedzic, "Optical trapping and manipulation of viruses and bacteria," Science 235(4795), 1517-1520(1987).

[3] D. G. Grier, “A revolution in optical manipulation,” Nature 424(6950), 810-816(2003).

[4] J. B. Wills, K. J. Knox, and J. P. Reid, “Optical control and characterization of aerosol,” Chem. Phys. Lett.

481(4-6), 153-165(2009).

[5] S. A. Tatarkova, A. E. Carruthers, and K. Dholakia, "One-Dimensional Optically Bound Arrays of Microscopic Particles,” Phys. Rev. Lett. 89, 283901(2002)

[6] A. T. O’Neil, I. MacVicar, L. Allen, and M. J. Padgett. "Intrinsic and Extrinsic Nature of the Orbital Angular Momentum of a Light Beam.” Phys. Rev. Lett.88, 053601(2002)

[7] C. Alpmann, R. Bowman, M.Woerdemann, M. Padgett, C. Denz. "Mathieu beams as versatile 
light moulds

for 3D micro particle assemblies”. Opt. Express. 18(25). 26084-26091(2010).

[8] Mike Woerdemann, Christina Alpmann, and Cornelia Denz. "Optical assembly of

microparticles into highly ordered structures using Ince-Gaussian beams”. Appl. Phys. Lett. 98,

111101(2011).

[9] Sandeep Tauro, Andrew Bañas, Darwin Palima and Jesper Glückstad. “Dynamic axial

stabilization of counter-propagating

beam-traps with feedback control”. Opt. Express. 18(17) 18218-18222(2010)

[10] Stephanie H. Jones,ab Martin D. Kinga and Andrew D. Ward. "Determining the unique refractive index properties of

solid polystyrene aerosol using broadband Mie scattering from optically trapped beads.” Phys.

Chem. Chem. Phys. 15, 20735(2013)

[11] L. Rkiouak, M. J. Tang, J. C. J. Camp, “Optical trapping and Raman spectroscopy of solid particles.” Phys. Chem. Chem. Phys. 16, 1142(2014).

[12] Yong-Le Pan,* Steven C. Hill, and Mark Coleman. "Photophoretic trapping of absorbing particles in air and measurement of their single-particle Raman spectra”. Opt. Express. 20(5).

5325-5334(2012) 